\title{
DIAGNOSTICS AND CORRECTION OF THE FUNCTIONAL STATE OF THE HUMAN ORGANISM WITH THE "CHARM"- DIAGNOSTIC COMPLEX
}

\author{
Nikolay D. Devyatkov, Vladimir Ya. Kislov, Vladimir V. Kolesov, Vladimir I. Grachev \\ Kotelnikov Institute of Radioengineering and Electronics of Russian Academy of Sciences, http://cplire.ru \\ Moscow 125009, Russian Federation \\ info@cplire.ru,kvv@cplire.ru,grachev@cplire.ru
}

\begin{abstract}
Absrtact. The therapeutic-diagnostic complex (TDC) is intended for electropuncture diagnostics and correction of the functional state of the organism with the help of puncture extremely high frequencies (EHF)-therapy. TDC allows to conduct studies of the electrophysical parameters of the skin as a function of time, to search for acupuncture points on the human body, diagnostics using the methods of Nakatani and Voll, and puncture EHF correction of a person's functional state. TDC is applicable for assessing the effectiveness of any type of treatment in the clinic, in express diagnostics in the course of medical examinations, medical examinations, medical care in medical institutions, in assessing the health status of people in everyday life.
\end{abstract}

Keywords: functional state of the human body, electropuncture, EHF-therapy, acupuncture points, electrophysical parameters of the skin, express diagnostics

UDC 538.573.61

Bibliography - 25 references

Received - 26.08.2018

RENSIT, 2018, 10(2):257-268

DOI: $10.17725 /$ rensit.2018.10.257

\section{CONTENT $^{1}$}

1. INTRODUCTION (257)

2. THE EQUIPMENT OF THE THERAPEUTICDiagnostic COMPlex "Charm" (259)

3. Methodological basis of Electropuncture DiAgNOSTICS (262)

4. Biophysical fundamentals of ELECTROPUNCTURE DIAGNOSTIC METHODS (263)

5. Some REsults of Clinical trials of TDC "ChaRM" (264)

6. Conclusion (266)

REFERENCES (267)

\section{INTRODUCTION}

At the present time, in connection with the development of methods of the system approach in medical diagnostics, reflexology, preventive medicine, valeology, methods of non-medicamentous influence, on the one hand, and with the development of methods of radio electronics, control theory and telecommunication and information computer technologies, on the other hand, it becomes possible

${ }^{1}$ This article was written in 2007 for the journal Millimeter Waves in Biology and Medicine (ZAO MTA-EHF), but due to the large volume it was not accepted for publication. task of monitoring, diagnosis, evaluation and correction of the functional state of the human body in real time.

Among the ranges of electromagnetic waves used in medicine as diagnostic and therapeutic tools, the range of extremely high frequencies has recently been widely used. The development of the physical foundations for the creation of equipment in the millimeter range was conducted under the guidance of Academician ND Devyatkov, starting with the 60-ies of the last century. Along with powerful sources for technical purposes, low-intensity solidstate sources of MM radiation based on avalanche transit diodes (ATD) and Gunn diodes were created, on the basis of which industrial samples of sources for medical use were developed [1].

The effectiveness of the biological effect of low-intensity electromagnetic radiation in the millimeter range as a physical factor for the construction of new medical equipment is justified by the fact that all terrestrial biological objects are protected by the atmosphere from exposure to solar radiation of the millimeter wavelength range. Therefore, biological objects, 
including humans, have increased sensitivity in this wavelength range. In laboratory studies for the first time it was experimentally found that low-intensity MM-radiation has a pronounced biological effect [2].

As a result of developments based on the results of numerous clinical trials, a whole gamut of therapeutic EHF-devices was created, for which licenses were issued by the Ministry of Health of the Russian Federation. The basis of all these devices, mass-produced at a number of industrial enterprises of the Russian Federation, are priority developments of the basic EHF-device type "Yav" based on ATD generators.

Perennial clinical experience of successful application of EHF-therapy has shown its effectiveness in affecting certain areas of the body, including. reflexogenic zones and acupuncture points. Electrophysical parameters of the skin in such zones and points are used in electropuncture techniques to diagnose the functional state of various systems and organs. Monitoring of the results of EHF-correction of the functional state of the human body was carried out with the help of a computerized electropunctural diagnostic complex.

In the Kotelnikov Institute of Radioengineering and Electronics of RAS of team of developers under the leadership of Academician N.D. Devyatkov and Professor V.Ya. Kislov established the therapeutic and diagnostic complex "CHARM" (TDC "CHARM"), intended for electropuncture diagnostics and correction of the functional state of the human body with the help of created EHFequipment [3]. The combination of electropuncture express diagnostics with puncture EHF-therapy made it possible to develop a method for assessing the effectiveness of treatment, control and correction of the functional state of the human body [4].

Based on modern methods of radioelectronics, the TDC "Charm" allows to perform an objective electropunctural express diagnostics of the functional state of the main internal organs of the human body using the method of riodoraku (based on the measurement of skin electrical conductivity in 24 representatively points), and the puncture EHF-correction of the imbalance of the functional state of the meridian system and bringing it to normal (hereinafter, the terminology adopted in eastern reflexotherapy is used).

At the heart of the worldview of ancient Chinese philosophy is the doctrine of "yin" and "yang." According to this teaching, all things and phenomena have two opposing sides, complementing each other, these are two complementary parts of the whole. Different relationships between yin and yang determine the different states of the surrounding world, including the state of the human body. According to Chinese medicine, the human body is also divided into two opposite functional parts. The concept of yang in the body include: the upper body, its surface, back, six "hollow" organs (stomach, large intestine, small intestine, gall bladder, bladder and so-called "triple heater" -functional system corresponding to the endocrine system). To the concept of yin are: the inner part of the body, the stomach, six "dense" organs, the so-called. organs of energy storage (heart, lungs, spleen, kidneys, liver, pericardium), blood.

For traditional Chinese medicine, the "organ" is not so much an organic structure, but mainly a functional system that is related to the physiological and psychological state of the organism. All organs of the yang interact with the yin organs and with each other according to cyclic laws (a large circle of circulation). This interaction takes place over a certain time cycle and along certain meridian lines. Interaction of meridians with each other individually and in the system as a whole ensures dynamic maintenance of homeostasis. Thanks to these interrelations, it is possible to conduct disease diagnostics, treatment and prevention of diseases. The disease in this model is considered as a violation of the harmonic balance between yang and yin, and the doctor's task is to diagnose and correct the state of the meridian system.

The behavior of the meridian system can be modeled by methods of nonlinear dynamics [5]. Numerical analysis of such a self-oscillating system makes it possible to obtain information on various states of the meridian system corresponding to normal and pathological states. 
Puncture correction is based on the principles of oriental reflexology medicine, which has ancient roots and millennial traditions, covers the study of the physiology of the human body, its pathology (the origin of diseases and their causes), methods of medical research, diagnosis, therapy and disease prevention. One of the main diagnostic methods in oriental medicine is pulsodiagnostics, and the therapeutic method is acupuncture. In this case, in comparison with western medicine, they treat not the disease, but the patient, i.e. improve the functional state of all systems of the body, its organs and defense mechanisms. The basis of the method of treatment is to adjust the condition of 12 main functional organs (meridians) - internal and external, by means of acupuncture, providing a stable dynamic homeostasis of the organism on the basis of a correct diagnosis.

According to traditional oriental medicine, the state of the biologically active point (acupuncture point) reflects the functional state of the corresponding organ, the active point is, as it were, the organ window into the external world. Deviations in the work of internal organs lead to changes (reflexes) of certain points on the surface of the body. Thus, there are many points with increased conductivity on the human skin, the location and degree of excitation of which clearly depends on the disease [6].

At present, low-intensity radiation of millimeter wavelength range $(4.9 \mathrm{~mm}, 5.6 \mathrm{~mm}$, $7.1 \mathrm{~mm}$ ) is used in EHF-therapy when exposed in reflexogenic zones. Low-intensity millimeter radiation refers to non-ionizing radiation. It in principle can not have a destructive effect on the cells of the body and therefore is safe. For more than 20 years of millimeter wave application in clinical practice it has been shown that a positive therapeutic effect is achieved in the treatment of a number of diseases, and there are practically no obvious contraindications. To date, we can name a significant number of various diseases in which the effectiveness of EHF-therapy has been proved: peptic ulcer and duodenal ulcer, pain syndromes, dermatology, hypertension, diseases associated with circulatory disorders in the brain and extremities, a number of oncological diseases, hematology, neurological diseases, gynecological diseases, postoperative conditions, ischemic heart disease, and others.

Stressing the effectiveness of EHF-therapy, it is possible to distinguish the following features of it: non-invasiveness of the method, use of EHFtherapy as monotherapy (ie, drugless therapy with a polytherapeutic effect, when treatment of a single disease are treats secondary diseases), lack of allergy to EHF- radiation, the presence of positive emotions in patients with treatment (pain sensations disappear, when exposed) [7].

\section{THE EQUIPMENT OF THE THERAPEUTIC-DIAGNOSTIC COMPLEX "CHARM"}

The main goal of developing a therapeutic-diagnostic complex (TDC "Charm") is the creation of new medical equipment that can replace the traditional technique of diagnostics and acupuncture, simplify acupuncture recipes, thereby ensuring its massig, including homey, use.

TDC "Charm" is a new computerized complex with diagnostic software, therapeutic recipes for equalizing pathological abnormalities and an extensive database. In this case, three systems are connected together in a single complex: expressdiagnostics of human body systems, the puncturing EHF-influence and a technique for correction of functional states of the main organs (EHF-puncture recipes).

The complex includes:

- portable personal computer of "Notebook" type, remote electronic unit with measuring electrodes.

- a software package that provides the user with a user-friendly interface that allows you to display all current information on the monitor, maintain a database, search, display information in the form of graphs and tables on the monitor and printer;

- therapeutic device for EHF therapy with an EHF-radiation indicator operating at fixed wavelengths of 5.6 and $7.1 \mathrm{~mm}$ and having a low power flux density $\left(10 \mathrm{~mW} / \mathrm{cm}^{2}\right)$;

- technical description and methodical manual.

The measuring part of the electronic board has a galvanic isolation from the patient's body along the power circuits through the information channels 


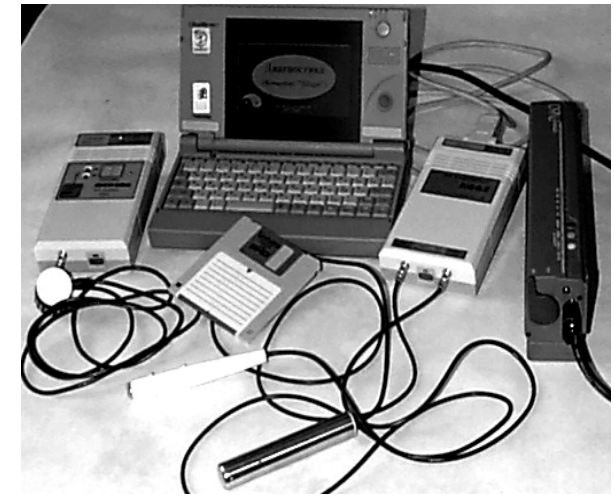

Therapeutic-diagnostic complex "Charm" for electropuncture express-diagnostics and EHF-therapy (version with autonomous power supply).

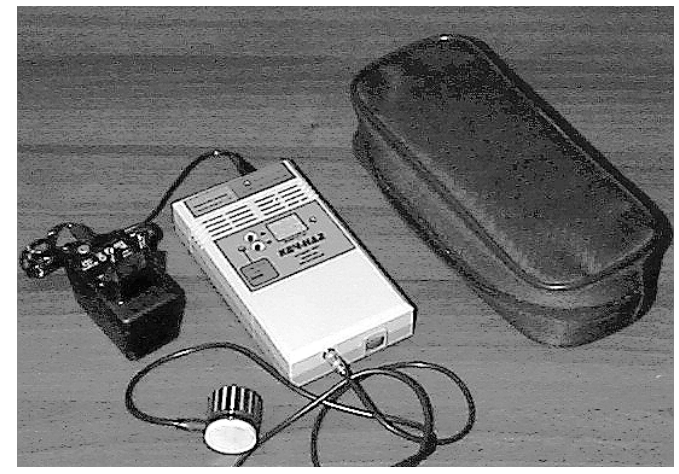

Apparatus "KVCH-ND2" of the new generation with autonomous power for puncture EHF-therapy (wearable version).

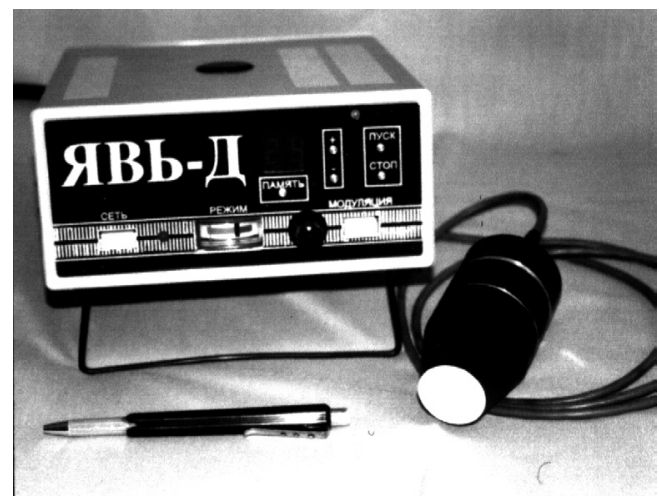

Apparatus "Yav-D" with a remote irradiation head for puncture EHF-therapy.

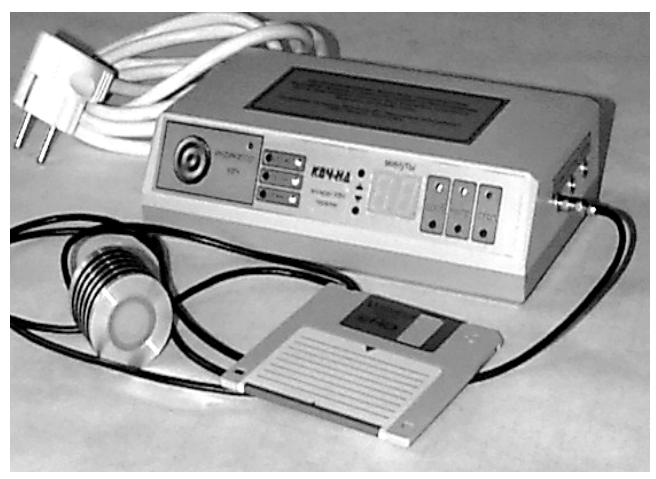

The device "KVCH-ND" for three frequency ranges 7.1: 5.6 and $4.9 \mathrm{~mm}$ for puncture EHF-therapy.

Fig. 1. Equipment for diagnosis and EHF-therapy.

from the computer and is therefore absolutely safe. This design allows you to work in the absence of mains supply (in the field, with seriously ill patients, etc.). In this case, complete information is stored in the database.

The complex allows

- produce searching for acupuncture points on the human body;

- conduct electropuncture diagnostics by the method of ryodoraku;

- conduct research on the electrophysical parameters of the skin as a function of time;

- conduct EHF-correction of the functional state of the internal organs of man.

In this case, the time of examination of one patient does not exceed 5 minutes.

As an example, the following areas of application of the complex can be named:

- express-diagnostics for the professional examination of work collectives, employees of firms, for the prophylactic examination of population groups, for the care of patients of polyclinics, hospitals, medical posts, in assessing the health status of family members, etc.,

- for therapeutic purposes to regulate bioenergetic and physicochemical processes in the tissues and systems of the human body,

- to evaluate the effectiveness of treatment (drug, surgical, radiation, etc.)

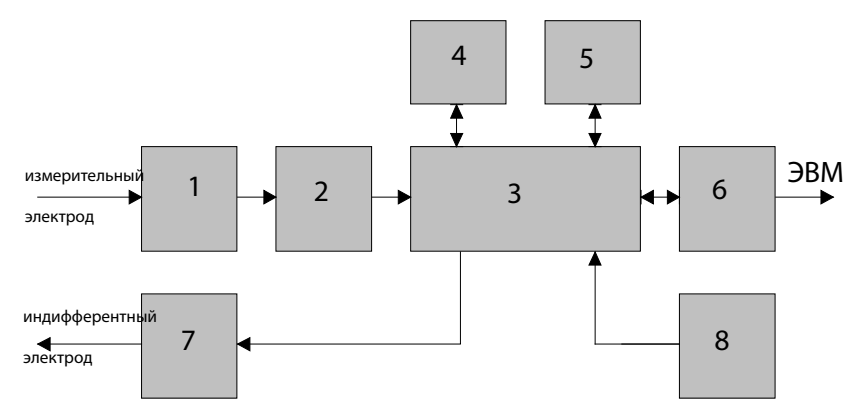

Fig. 2. Block diagram of the measuring unit TDC Charm: 1-input converter, 2 - analog-digital converter, 3 - microprocessor, 4 - random access memory, 5 - read-only memory, 6-optocoupler block, 7 - reference voltage block, 8 - rechargeable battery. 

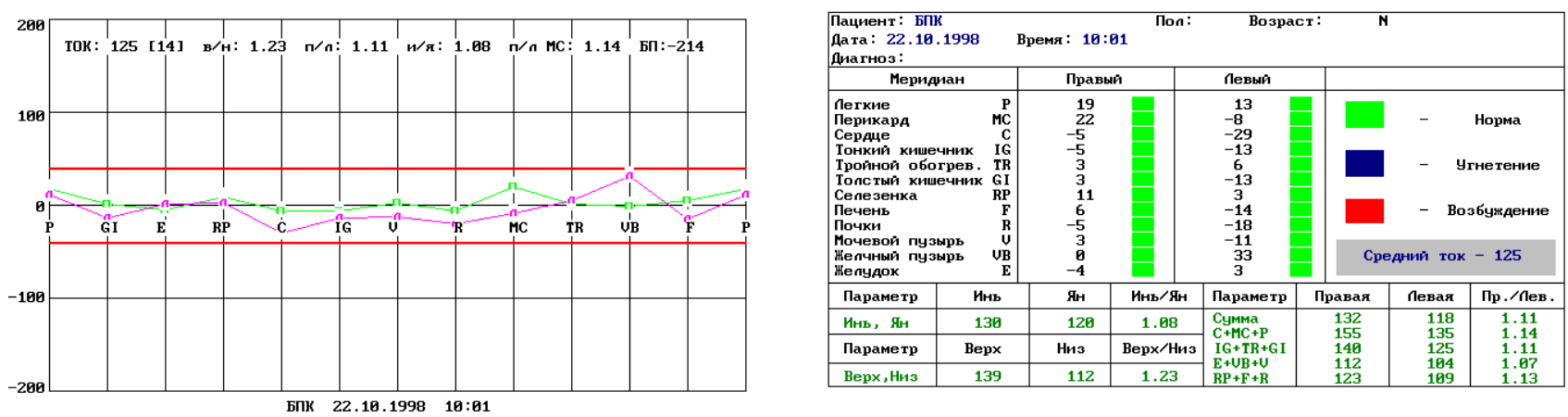

Fig. 3. Diagram and table of the functional state of a practically healtby person. The red lines ( \pm 40$)$ limite the normal range.

Simplicity in handling and management of the complex makes it possible to apply it not only in medical institutions, but also in the home. The complex allows rapid diagnosis and correction of 12 major functional systems of the body-lungs (P), vascular system (MC), heart (C), small intestine (IG), endocrine system (TR), spleen / pancreas (RP), liver $(\mathrm{F})$, kidney $(\mathrm{R})$, bladder $(\mathrm{V})$, gallbladder (VB), large intestine (GI) and stomach (E) - according to the electropuncture conductivity condition of meridians in 24 representative points (ryodoraku method) [8], as well as assess the condition of individual human organs (or parts thereof) according to the Foll method [10], in which parameters of the transient process are measured after a dosed low-power signal is supplied to biologically active points. Combination of the method of ryodoraku and elements of the Voll method is most optimal.

In Fig. 1 shows the developed equipment for diagnosis and EHF-therapy. In Fig. 2 shows the block diagram of the measuring unit TDC "Charm". In Figs. 3-7 shows the diagnostic maps of a healthy person, an example of EHF correction of a functional state and various pathological conditions.

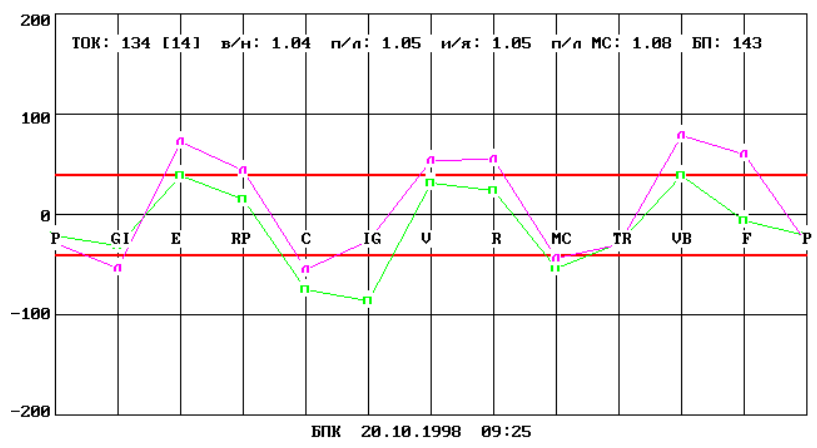

TDC "Charm" was approved in the institutions of the Ministry of Health of the Russian Federation and medical institutions of the Ministry of Defense of the Russian Federation. Clinical trials were conducted according to the decision of the Committee for New Medical Technology of the Ministry of Health of the Russian Federation. The conducted tests showed the effectiveness of the TDC "Charm" in the treatment of various groups of diseases.

At the same time, positive recommendations were given on the application of TDC "Sharm" for the treatment of stroke, diabetes, trigeminal neuralgia, osteochondrosis, hypertension, etc., as well as a more general methods has been formulated for those cases where conventional medical treatment is used. For example, the puncture EHFtherapy, performed with the help of TDC "Charm" as part of complex treatment in patients with complicated osteochondrosis of the spine, allows, in comparison with the control group in a shorter time and completely to stop pain syndromes, to restore the volume of active movements in the joints and sensitivity of the skin in the corresponding zones of innervation.

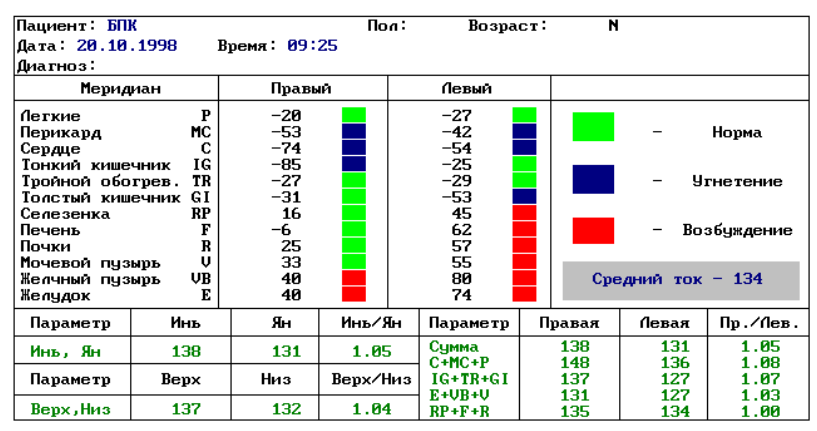

Fig. 4. Diagram and table of a patient's functional state before EHF-correction. The red lines ( \pm 40$)$ limite the normal range. 

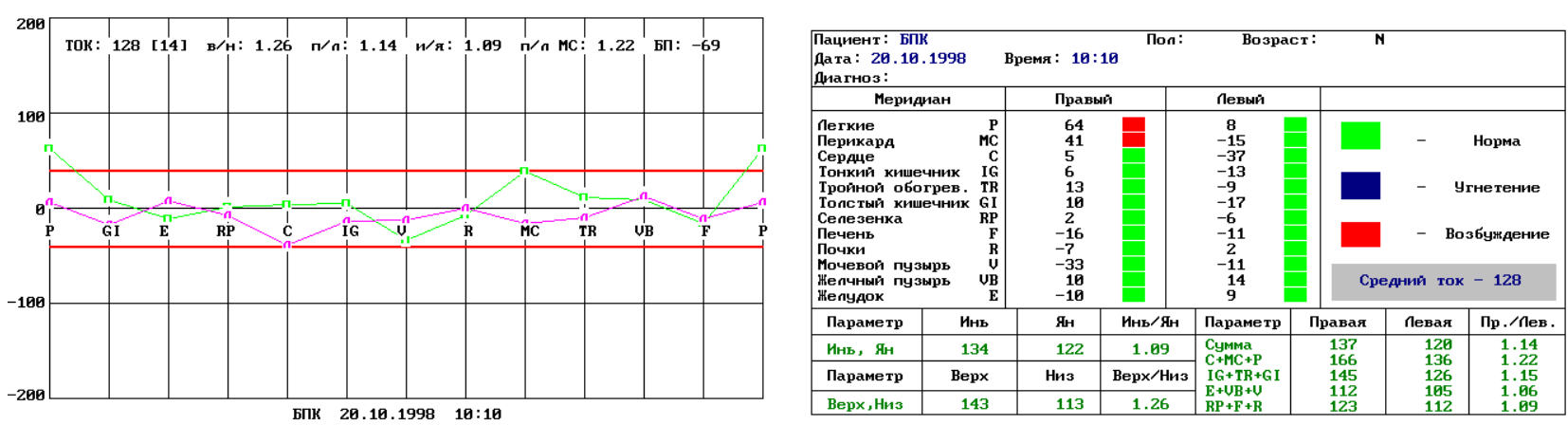

Fig. 5. Diagram and table of a patient's functional state after EHF-correction. The red lines ( \pm 40$)$ limite the normal range.

In the treatment of type 2 diabetes mellitus, there was a significant reduction in the glucose level in the blood of patients with fasting. In patients with consequences of the violation of cerebral circulation and hemiparesis, a decrease in spasticity on the side of hemiparesis and a normalization of blood pressure were observed. In patients with hypertensive disease of the first stage after the application of the puncture EHF-therapy in a complex of therapeutic measures, the clinical recovery came quite quickly and effectively. During the clinical trials of side effects and complications of EHF-puncture therapy, applied in accordance with the above method, was not observed.

\section{METHODOLOGICAL BASIS OF ELECTROPUNCTURE DIAGNOSTICS}

Virtually all processes in the body are accompanied by a change in the electromagnetic characteristics, which can be fixed by various contact and noncontact physical methods. One such method is electropuncture diagnostics, based on the measurement of electrocutaneous characteristics [10].

The most common and currently promising electropuncture techniques (Nakatani, Voll, auricular, etc.) measure the skin-galvanic reaction by means of

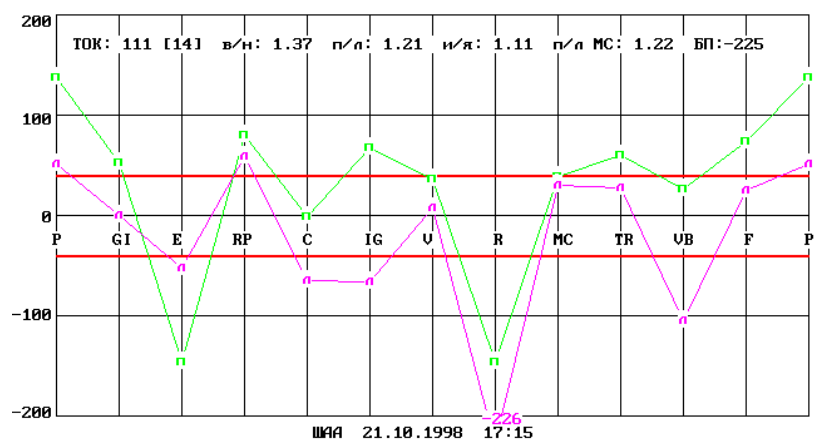

contact metal electrodes with a probing voltage of a certain sign (in the Nakatani technique negative, Voll-positive). This ensures the stability of the contact resistance in the contact area of the skin with the measuring electrode [11, 12]. Electropuncture techniques are based on empirically selected parameters of the electrical measuring circuit.

In all electropuncture diagnostic measurements, the skin is a transitional element for the external measuring circuit. As is known, the skin has sensitivity in a wide range of the electromagnetic wave spectrum [13], carrying out active energy and information interaction of the organism with the external environment. As an organ, the skin is one of the oldest biosystems of the body, from the simplest to the mammals and man. Apparently, the system of autoregulation of various biochemical processes occurring in the skin is laid still at the embryonic stage of the development of the human body, and at the initial stage has a rather simple structure, which in turn is organically linked with nearby rudiments of various organs and parts of the body, and then, developing with the body, takes a complex, ramified character [14]. Thus, it is possible to explain the appearance of reflexogenic reactions in certain places on the skin when exposed to various internal organs.

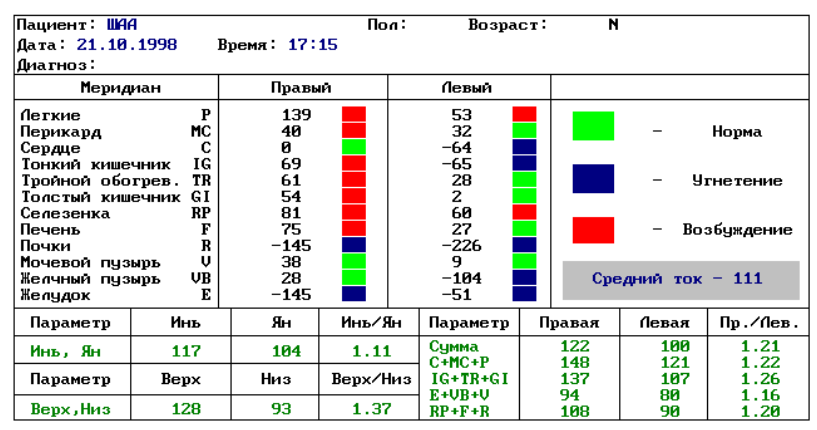

Fig. 6. Diagram and table of the functional state of a patient with chronic diseases of the stomach, kidneys and gallbladder. 

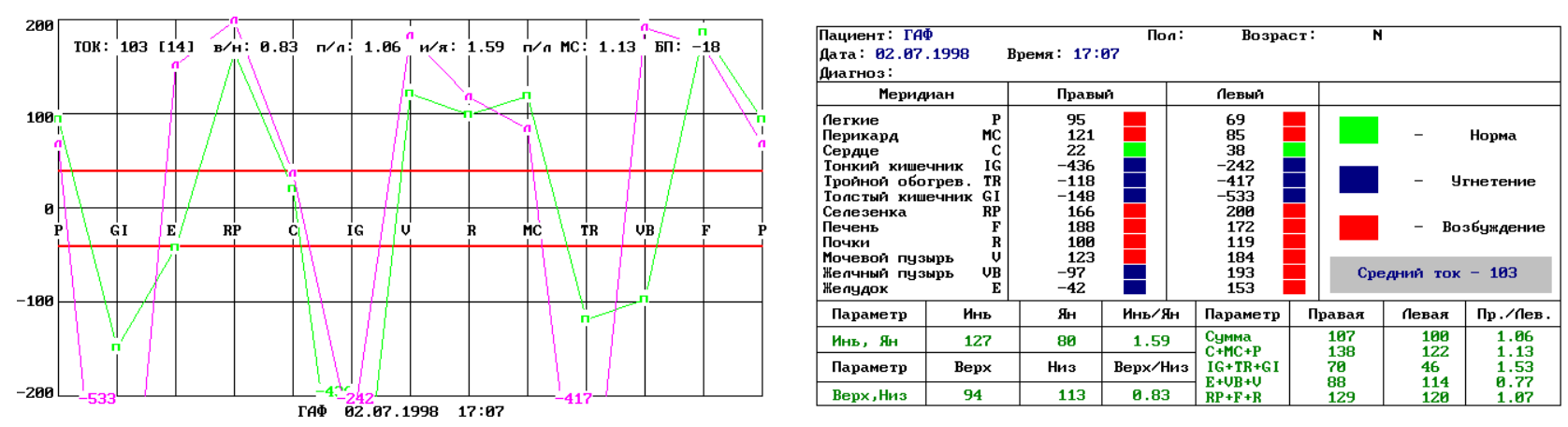

Fig. 7. Diagram and table of the functional state of a seriously ill patient.

The skin has its own system of autoregulation, which, apparently, is neurohumoral in nature. The epidermis of the skin and the epithelium of the digestive tract are two tissues most prone to direct influences from the outside world. In both tissues, mature differentiated cells are found in the most vulnerable areas, are quickly worn out and are also quickly replaced as a result of the proliferation of less differentiated cells in more protected areas.

Liquid media are in their enclosing volumes, bounded by surfaces with different properties. The permeability of these surfaces for different components of liquid media is different and variable, provides a state of dynamic equilibrium. At the same time, the water and electrolyte balance are functionally inseparable [15].

The primary condition of normal vital activity of the body cells (and skin cells in particular) is the constancy of the ionic composition and $\mathrm{pH}$ of biological fluids. Preservation of the constancy (homeostasis) of differences in the electrolyte composition of blood plasma and extracellular fluid, on the one hand, and intracellular fluid, on the other, is one of the defining properties of the body's regulatory systems.

Thus, the diagnosis of the functioning of metabolic processes in the skin can serve as the basis for biomedical diagnostic techniques.

\section{BIOPHYSICAL FUNDAMENTALS OF ELECTROPUNCTURE DIAGNOSTIC METHODS}

If the monitoring of metabolic processes in the skin is taken as the basis of the method of functional diagnostics, then the problem is in the first approximation reduced to measuring the electrophysical characteristics of that electrolyte medium that directly participates in metabolic processes at the cellular level through cell membranes in the active layers of the epidermis and dermis.

The upper layers of the epidermis are densely lying keratinized cells with a low water content and have a relatively high electrical resistance. In direct contact with a metal measuring electrode, almost all the probe voltage is applied to a relatively small area between the electrode metal and the lower layers of the epidermis, which are saturated with an intercellular fluid, which is a strong electrolyte of a complex chemical composition.

Thus, the measured conductivity of the transition region consists of conductivity of the high-resistance upper layers of the epidermis and the intrinsic conductivity of the cells of the intermediate layers of the epidermis. In this case, it is necessary to take into account not only the layered structure of the epidermis and the dermis, but also the porosity of the various skin areas, which determines its excretory and mechanoreceptor properties. It is also known that in the field of biologically active points, the skin has increased looseness and saturation with the intercellular fluid [16].

Thus, in order to increase the information, and therefore the diagnostic value of the method, it is necessary to isolate from the integral conductivity of the entire measured circuit the electrophysical characteristics of the biologically active layers of the epidermis and dermis.

The model representation of the measuring electrical circuit in the first approximation should include the electrophysical characteristics of the layers of the skin cells themselves, and also take into account the shunting action of the porous 
cellular structure, which, when filled with electrolyte, can make significant changes in the quantitative relationships.

\section{SOME RESULTS OF CLINICAL TRIALS OF TDC "CHARM"}

The examination and treatment was carried out in three groups of patients:

1. diabetes mellitus type 2 (insulin-independent),

2. hypertensive disease of the 1 st stage and neurocirculatory dystonia according to the hypertonic type,

3. osteochondrosis of the spine with radicular syndrome.

The examination of patients with the help of TDC "Charm" followed by EHF-puncture therapy of revealed abnormalities was carried out, usually in the morning hours on an empty stomach, or 2 hours after eating or carrying out any other procedures. Points of influence were chosen according to the rules of traditional oriental acupuncture. The initial information for the choice of impact points was tabulated and graphical data obtained as a result of conducting electropuncture diagnostics with the help of TDC "Charm". The method of tonification of the "oppressed" meridians was used as the main method of influence. In those cases quite often encountered, when there was a friendly change of several meridians (two, three) at the same time, the so-called group lo-points were used for the therapeutic effect.

The results of the impact were taken into account by repeated electropuncture diagnostics after 1-1.5 hours and after 1 day; For one exposure session, two to five points were used, the selection of which was carried out individually, depending on the diagnostic results.

The clinical picture of diabetes was first described almost 2000 years ago by the Roman philosopher and physician Celsus, but the problem of cure for him has not been resolved to this day. From the moment of detection of the disease, the patient, as a rule, is attached to the sugar-reducing drugs for the rest of his days and is exposed to their side effects. An important actual problem is the reduction, with other equal conditions, of the dose of the drugs used due to physical methods of treatment and, in particular, EHF-therapy.
12 patients ( 9 men and 3 women aged 39-56 years) with type 2 diabetes mellitus were examined and treated with the help of the "Charm".

Eight patients had complications of the disease in the form of diabetic polyneuropathy, angiopathy of the vessels of the kidneys, retina of the eyes, lower limbs. All patients had concomitant diseases: peptic ulcer disease of the duodenum (4 persons); chronic ischemic heart disease (5 people); urolithiasis (2 people); consequences of transferred serum hepatitis (1 person). The duration of the disease, confirmed by medical documents, ranged from 1 year to 15 years. All patients were on treatment in a specialized department and received complex therapy: maninil, vascular, antisclerotic, trophic drugs, antihypoxants, vitamins, symptomatic agents, treatment with hyperbaric oxygenation.

All patients had a significant deviation of the diagnosed parameters from the norm, which, as a rule, were multidirectional in different meridians. At the same time, it should be noted that the manual meridians (IG, TR and GI) were in a state of "oppression." For weakened patients with low mean current for the first treatment session used point RP21-symmetrically, for 5-6 minutes on each side. In subsequent sessions MC5, TRS, V65, RP6, E36, and less frequently GI11, VB34, etc. were used more frequently. The time of exposure on the point depended on the degree of "depression" of the meridians and was 3-4 minutes at the exponent (11.5) $\mathrm{N}$ up to $8 \mathrm{~min}$ at the exponents greater than 2 N. The total exposure time for one session was to 30 minutes.

When comparing changes in the level of glucose in the blood of fasting patients in the group receiving EHF-therapy and in the control group, it is evident that in the first there is a distinct decrease in blood glucose from $14.0 \pm 8.2 \mathrm{mmol} / 1$ to $6.6 \pm 1.8 \mathrm{mmol} / 1$ on average group for two weeks, while in the control group there were only fluctuations of this averaged index in the range of $7-11 \mathrm{mmol} / 1$ with a weak tendency to decrease in the first two weeks of stay in hospital.

This is clearly seen in Fig. 8, which shows the dynamics of fasting blood sugar, depending on the duration. Herewith, the differences between the compared groups at the end of the treatment course were statistically significant $(p<0.05$ by the 
Wilcoxon test), which proves the effectiveness of the EHF-therapy.

Thus, it was shown that EHF-puncture therapy can be recommended in the complex treatment of type 2 diabetes mellitus, since, without exerting side effects on a person in this way of administration, at the same time contributes to a faster and more stable decrease and normalization of glucose in blood of patients [18].

The importance of the problem of treating patients with essential hypertension is still not reduced, which is due to the wide spread of the disease all over the world, and the severe consequences to which it leads. The constant intake of drugs is often accompanied by serious complications, in connection with which the possibility of applying EHF-puncture therapy is very relevant.

A clinical examination of 18 patients with stage I hypertensive disease and neurocirculatory dystonia was carried out according to the hypertonic type, accompanied by cephalgia, cardialgia and other asthenoneurovalic symptoms (6 women and 12 men aged 20 to 46 years).

In addition to blood pressure indicators, central hemodynamic parameters were studied in all patients: minute volume of blood circulation $(\mathrm{ml} /$ $\mathrm{min})$, pulsatile volume $(\mathrm{ml})$, heart rate ( $\mathrm{min})$.

The control group consisted of 20 hypertensive patients of the 1 st stage and neurocirculatory dystonia according to the hypertonic type, who received drug therapy according to indications.

EHF-therapy was carried out by the apparatus "Yav". Time and conditions of the procedure are the same as in the previous group on diabetes. When conducting electropunctural diagnostics in all patients, the inhibition to some extent of

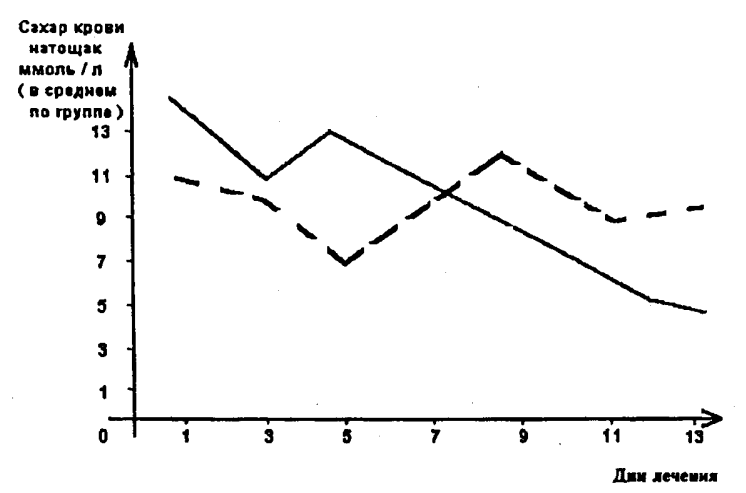

Fig. 8. Dynamics of fasting blood sugar in a group of patients with diabetes mellitus (solid curve-group receiving EHF-therapy, dashed-control group). the upper meridians (IG, TR, GI) and a slightly higher average current was found than in groups of patients with other diseases. The course of treatment consisted of ten procedures. The points for exposure were chosen solely from the results of a diagnostic study in an amount of two to four points per session with a total duration of 12 to 30 minutes. The most frequently used points were TR8, MC, on both sides, as well as points GI4, E36.

In all patients, as a result of the treatment, there was a decrease in arterial pressure - systolic and diastolic to normal indications; decrease in heart rate. The minute volume decreased on average from $4.21 \pm 0.37$ to $3.8 \pm 0.51$. The dynamics of arterial pressure in the compared groups is clearly shown in Fig. 9.

Thus, after the application of EHF-punctures in a complex of therapeutic measures for this nosology, clinical recovery has come quite quickly and effectively. TDC "Charm" provides the possibility of objectifying and visualizing the process of treatment and especially dispensary observation of the patients in dynamics [18].

The next group consisted of patients with complicated cervical and lumbosacral osteochondrosis: 4 women and 6 men aged 32 to 46 years with a disease duration from 3 to 15 years. In seven cases, the pain syndrome was caused by the inflammatory process (radiculitis, ganglionevrit), and in three cases it was discogenic. In both ancient and European medicine, at this pain syndrome marks the appearance of points that are painful at palpation and have diagnostic or therapeutic value. Based on this, the points of general action E36, GI4, MC6,

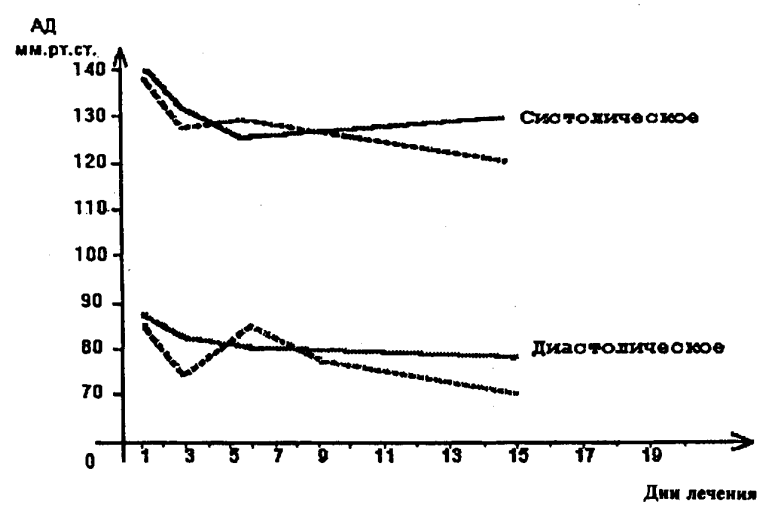

Fig. 9. Dynamics of arterial pressure in patients with NDC and bypertensive disease (dashed curve-group receiving EHF-therapy, continuous-control group, 1-systolic pressure, 2-diastolic pressure). 
RP6, TR8, V60 and the point of meridian of the spleen/pancreas and the posterior-median meridian were used in the treatment of this category of patients. Each patient received ten sessions of EHFtherapy with a total duration of the session from 16 to 30 minutes. In addition, patients received physiotherapeutic and medicamental treatment. In the control group, EHF-therapy was not performed. Criteria for the effectiveness of treatment were: the dynamics of reducing the intensity of pain and the timing of their elimination, restoration of skin sensitivity and the volume of movement of the joints. The intensity of pain was determined subjectively by a 4-point system: severe pain - 3 points, moderate - 2 , weak - 1 , no pain - 0 points.

In the group of patients who received EHFtherapy, the pain syndrome was completely eliminated in all the treated patients by the 11th day. In parallel, they recovered the volume of active movements in the joints and restored the sensitivity of the skin in the corresponding zones of innervation. The average dynamics of treatment of this group of patients and the control group is shown in Fig. 10. For diagnostic signs, EHF correction leads to statistically reliable normalization of the balance of meridians, corresponding to the time for clinical recovery.

As a typical example, one can consider the case of a patient P. (36 years old), hospitalized for emergency indications with the diagnosis of "ganglionevritis with severe pain syndrome. Cervical osteochondrosis". After two sessions of EHF-therapy (the points of the collar zone, as well as V43, V60, IG9, IG10, TR8, E36, GI4, GI11 were used), decreased pain in the neck, right shoulder, increased volume of active

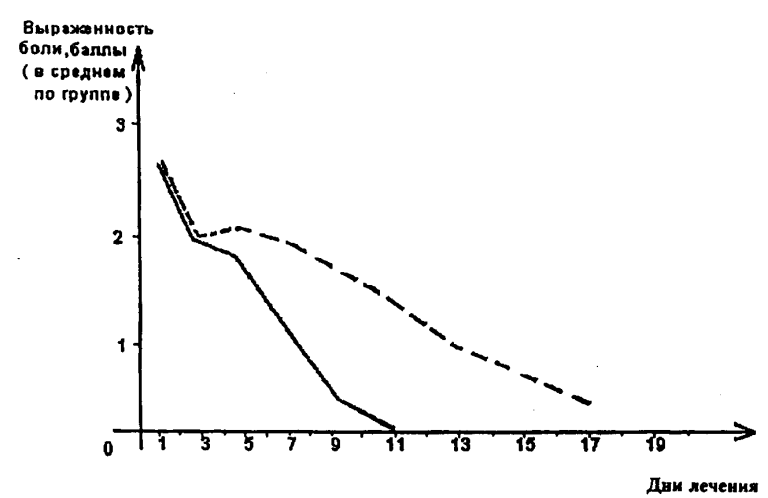

Fig. 10. Dynamics of pain syndrome changes in patients with osteochondrosis (solid curve-group receiving EHF-therapy, dashedcontrol group). movements in the cervical spine. After the seventh session, only minor pains remained in the area of the right shoulder, which completely stopped after the tenth session. The sensitivity of the skin and the volume of active movements in the cervical spine and shoulder joint were completely restored.

With electropuncture diagnostics after the conducted course of EHF-therapy, the normalization of diagnostic indices attracts attention. Normalization was characterized by a high degree of reliability for the entire group of patients. On average, in the group of patients who received EHF-therapy, the pain syndrome was completely eliminated and the volume of active movements was restored by 11th day. In the control group, similar indicators were achieved by day 17 and only partially. Thus, we can conclude that TDC "Sharm" is highly effective in diagnosing and treating patients with complicated osteochondrosis of the spine with severe pain syndrome and can be successfully used both in hospitals and in polyclinics [18].

\section{CONCLUSION}

Thus, it is shown that the therapeutic-diagnostic complex "Charm" has wide reflex-therapeutic possibilities:

- Express diagnostics by the method of ryodoraku;

- measurement of the characteristics of transient processes by the Foll method;

- exposure to low-intensity EHF-radiation for the purpose of correcting the functional state of individual organs, systems and the entire human body.

With the help of the "Charm" apparatus direct electrophysical measurements demonstrated the existence on the cutaneous surface of the human body of well conducting lines that coincide with the classical meridians [18]. On the basis of the reflexotherapy complex, it is also possible to study the phenomena associated with electropunctural manifestations of homeostasis, to study the complex system of points of acupuncture and meridians [19]

Clinical trials of TDC "Charm" have shown its effectiveness in the treatment of a wide range of diseases and, in particular, diabetes, cerebral circulation disorders [20], neurocirculatory dystonia, hypertension, osteochondrosis [17], inflammation of the trigeminal nerve [20], as well as many other diseases. TDC "Charm" has proved effective in 
diagnosing surgical pathologies and the process of rehabilitation in the postoperative period [22], in the diagnosis of psychophysiological [23] and stress conditions [24].

With the introduction of LDC "Sharm" and new technologies based on it, in mass medicine a significant social and economic effect can be obtained [25].

\section{REFERENCES}

1. Golant MB, Vilensky RL, Zyulina EA, etc. A series of wide-range low-power generators of millimeter and submillimeter wavelengths. PTE, 1965, 4:136-139 (in Russ.).

2. Devyatkov ND, Golant MB, Betsky OV. Millimeter waves and their role in life processes. Moscow, Radio i svyaz Publ., 1991, p. 169.

3. Devyatkov ND, Kislov VY. Computerized electrophysical diagnostics and EHF correction of the functional state of internal organs of a person. Radiotekhnika i elektronika, 1994, 39(12):2059 (in Russ.).

4. Devyatkov ND, Kislov VY, Kolesov VV, Smirnov VF, Otchertsov AV. Therapeutic-diagnostic complex "Charm". Coll. reports "Millimeter waves in medicine and biology". Moscow, IRE RAS, 1995, p. 178-179.

5. Belyaev RV, Kislov VY. Multi-loop autooscillation system with delay as a model of the system of acupuncture points of the human body. Radiotekhnika i elektronika, 1999, 44(11):123 (in Russ.).

6. Luvsan Gavaa. Methods of oriental reflexotherapy. Novosibirsk, Science, 1991.

7. Belyi YuN, Kislov VY, Kolesov VV, Smirnov VF, Teodorovich SL. Some applications of computerized diagnostics and EHF correction of the functional state of internal organs of a person. Col. reports "Millimeter waves in medicine and biology". Moscow, IRE RAS, 1995, p. 74-76 (in Russ.).

8. Hyodo MD. Ryodoraku treatment and operator approach to acupuncture. Osaka, Japan, 1975.

9. Voll R. Topographic Positions of the Measurement Points in Electroacupuncture According to Voll. Vol. 1-4. Uelzen, ML-Verlags, 1977.

10. NiboyetJEH. Le traitement des algiesparl'acupuncture. Paris, Maisonneuve, 1979.
11. NakataniY, Yamashyta K. Ryodoraku Akupunkture. Tokyo, 1977.

12. Voll R. Kopfherde, Diagnostik und Therapie mittels Elektroakupunktur und Medikamententestung. Uelzen, Med.-Lit. Verlag, 1974.

13. Berezovsky VA, Kolotilov NN. Biophysical characteristics of buman tissues. Kiev, Naukova Dumka, 1990.

14. Falin LEE. Atlas on normal histology and embryology. Moscow, Medgiz Publ., 1957.

15. Semenov NV. Biochemical components and constants of liquid media and human tissues. Moscow, Medicine Publ., 1971.

16. Fiore MSH. Atlas of human histology. Philadelphia, 1957.

17. Devyatkov ND, Gulyaev YuV, Belyi YuN, Kislov VY, Kislov VV, Teodorovich SL, Vasin IY, Polyanskaya LN, Kolesov VV, Smirnov VF, Chigin EP. Electrophysical fundamentals and clinical applications of diagnostics and EHF correction of human functional states. Radiotekhnika $i$ elektronika, 1995, 40(12):18871899 (in Russ.).

17. Aksenov AV, Grachev VI, Kolesov VV, Smirnov VF. Study of the electrical conductivity and potential of the skin for medical diagnosis. Abstracts of the II Congress of Biophysicists of Russia-1999, Moscow, 1999, p. 639-640.

18. Devyatkov ND, Grachev VI, Kislov VV, Kislov VY, Kolesov VV. Monitoring and maintenance of body homeostasis using electrophysical methods. Theses of the 55th session of the RNTUES named after. A.S. Popov "Radioelectronics and Communication at the Turn of the Millennium". Moscow, 2000, p. 228-229.

19. Devyatkov ND, Belyi YuN, Vasilenko AM, Kislov VY, Kislov VV, Kolesov VV, Smirnov VF, Chigin EP. The use of new reflexotherapeutic methods on the basis of the "Charm" therapeuticdiagnostic complex in the treatment of patients with cerebral circulation disorders. Millimeter waves in biology and medicine, 1996, 8:5-16 (in Russ.).

20. Megdyatov RS, Arkhipov VV, Kislov VY, Kolesov VV, Smirnov VF. The use of the therapeutic and diagnostic complex "Charm" in the complex therapy of neuralgia of the ternary nerve. Millimeter waves in biology and medicine, 1995, 5:20-24 (in Russ.). 
21. Barannikov AS, Belyi UN, Grachev VI, Kislov VYa, Kolesov VV, Panchenko IP, Smirnov VF. Electropuncture diagnostics of surgical pathologies and EHF-therapy of the rehabilitation process in the postoperative period. Millimeter waves in biology and medicine, 1999, 2:37-44 (in Russ.).

22. Barannikov AS, Belyi UN, Grachev VI, Kislov VYa, Kolesov VV, Panchenko IP, Smirnov VF. Restoration of psychophysiological functions in the postoperative period with the help of radiation from the EHF-range. Biomedical radio electronics, 1999, 2:49-55 (in Russ.).

23. Belyi YuN, Grachev VI, Kislov VV, Kislov VYa, Kolesov VV. Electrophysical monitoring of the states and their correction by low intensity millimetric radiation. Proc. 12th Int. Crimean Conf. "Microwave \& Telecommunication technology" (Crimico'2002), Sevastopol, Crimea, Ukraine, 2002, p. 67.

24. Belyi YuN, Kolesov VV, Chigin EP. EHFtechnology - as an element of telemedicine. Transactions 14 Ross. simp. with Int. participation "Millimeter waves in medicine and biology". Moscow, 2007, p. 75-78. 